\title{
MAX BAUMANN
}

\section{Weg vom Text \\ oder: Plädoyer für einen breiteren \\ Weg vom Text zum Verstehen}

\section{Einleitung}

Vor rund 500 Jahren fegte ein Bildersturm über Europa hinweg, der unschätzbares Kulturgut vernichtet hat. Ich beabsichtige nicht, mit einem "sola pictura" eine ebenso zerstörerische Gegenbewegung auszulösen. Aber ich bin der festen Überzeugung, dass die Zeit von "sola scriptura"1 auch im Recht endgültig abgelaufen ist, und dass es deshalb höchste Zeit ist, sich mit neuen Kommunikationsformen auseinanderzusetzen.

Nur das veröffentlichte Gesetz ist verbindlich - lex non obligat nisi promulgata -, wussten schon die alten Römer. Der moderne Rechtsstaat gerät ernsthaft in Gefahr, diesen grundlegenden Gedanken des Rechts zu verletzen, wenn er glaubt, in einer multimedialen Umwelt (fast) nur mit dem Medium der Schrift auskommen zu können. Tatsache ist, dass die Schreib- und Lesekompetenz in Konkurrenz zu anderen Kommunikationskompetenzen an (einer wohl ohnehin überschätzten) Bedeutung verloren hat (und weiter verliert, vgl. unten III.). Will der Staat seine Bürgerinnen und Bürger erreichen - im demokratischen Rechtsstaat unabdingbar - wird er nicht darum herum kommen, sein eigenes Kommunikationsverhalten demjenigen der Adressaten anzupassen: Allgemeinverbindlichkeit kann nur einfordern, wer auch in allgemein üblichen Formen kommuniziert.

\footnotetext{
${ }^{1} \mathrm{Zu}$ beachten ist auch, dass die Übergänge zwischen Schrift, Notation und Bild immer schon fließend waren. James Elkins schreibt dazu: „I do not think that we are in possession of a cogent and relevant concept of either term (word and image), and that lack can make word-image studies curiously ineffectual." Dementsprechend teilt er das Feld der "Grammae" in drei sich überlappende Felder: writing, notation and picture. Siehe dazu JAMES ELKINS, The Domain of Images. Ithaca 1998, $82 \mathrm{ff}$.
} 


\section{Ein Test}

A

1. Georg und Gerda sind die Eltern von Karl.

2. Hans ist mit Johanna verheiratet; ihr Sohn heißst Franz.

3. Peter ist der Sohn von Franz und Susanne.

4. Tinas Eltern heißen Hans und Johanna.

5. Werner und Lotte haben eine Tochter: Susanne.

6. Anna ist die Tochter von Karl und Tina.

7. Karl und Tina sind die Eltern von Karin.

8. Maria ist die Tochter von Franz und Susanne.
B

$$
\text { Vater Mutter Sohn Tochter }
$$

1. Georg Gerda Karl

2. Hans Johanna Franz

3. Franz Susanne Peter

4. Hans Johanna Tina

5. Werner Lotte Susanne

6. Karl Tina Anna

7. Karl Tina Karin

8. Franz Susanne Maria

Im Umgang mit juristischen Sachverhalten geübte Testpersonen (JusStudenten, Mitglieder des Sprachdienstes der Schweizerischen Bundeskanzlei, ein Auditorium von Fachleuten) sind nicht in der Lage, anhand der Datenvorgaben unter A oder B die gestellten Fragen innerhalb von 90 Sekunden zu beantworten.

Mit der folgenden Vorlage $C$ schaffen es aber praktisch alle, die gestellten Fragen in der gleichen Frist richtig zu beantworten. ${ }^{2}$

\footnotetext{
2 Das Diagramm C und damit die Idee für diesen Test stammt von WOLFGANG SCHNOTZ, Wissenserwerb mit logischen Bildern, in: BERND WEIDENMANN (Hrsg.), Wissenserwerb
} 
C

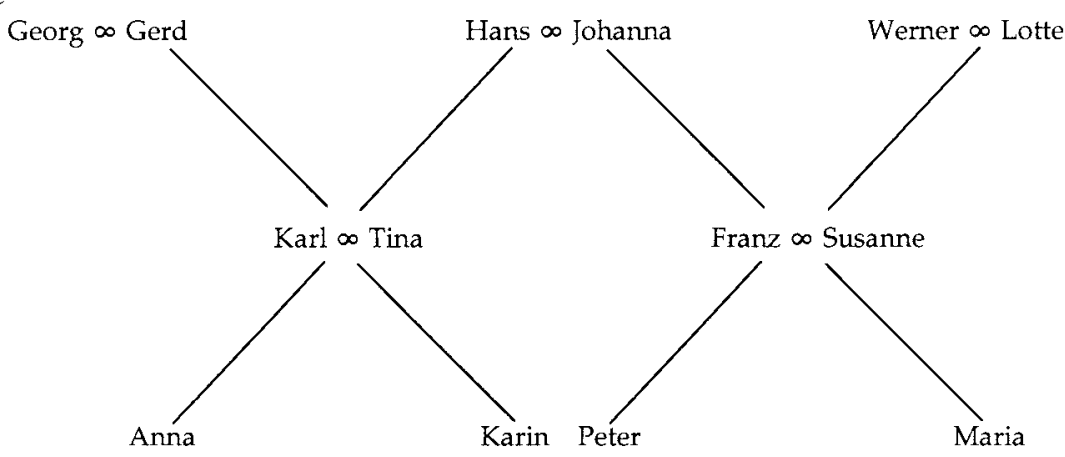

Ich bin mir im Klaren, dass diese kleine Demonstration nicht jede und jeden sofort zu einem glühenden Apostel der Bildverwendung bekehren wird. Es genügt, wenn vorstellbar geworden ist, dass bildliche Darstellungen gerade auch juristischer Sachverhalte zumindest als hilfreiche und Klarheit schaffende Ergänzungen zum Text gesehen werden dürfen. Man stelle sich z. B. den Nutzen eines Behörden-Organigrammes vor, das auf einer einzigen Seite Strukturen aufzeigt, deren verbale Darstellung ein Dutzend und mehr Seiten erfordert. Oder - um noch ein weiteres Beispiel zu bringen - an die Prozesstheorie aus der fertigenden Industrie angelehnte Verfahrensdiagramme können äußerst instruktive "Bilder" über die Wege und Sackgassen eines juristischen Prozesses aufzeigen. Doch zurück zum Titelthema:

\section{Weg vom Text}

1. Weg vom geschriebenen Text - weil der Text (als Schrift) allein nicht unbedingt zum Verstehen führt, ja sogar vom Verstehen wegführen kann. Die folgenden Ausführungen bewegen sich im Rahmen der hier nicht mehr weiter diskutierten Rahmen-Annahmen, dass

a) juristische Texte grundsätzlich schwierige Texte sind, nicht zuletzt deswegen, weil sie immer mehrfach adressiert sind; 3

mit Bildern. Bern 1994, 95 ff. Die Textfassung A und die hier verwendete Tabelle B stammen vom Autor.

${ }^{3}$ Vgl. dazu MARKuS NuSSBAUMER, Von Schwärmern und Skeptikern und ein Versuch, Realist zu sein. Bilanz und Entwurf des Sprachspiels vom unverständlichen Gesetz, in: KENT D. LERCH (Hrsg.), Recht verstehen. Verständlichkeit, Missverständlichkeit und Unverständlichkeit von Recht. Berlin 2004, 285-295. 
b) es im demokratischen Rechtsstaat Rechtstexte gibt und geben muss, ${ }^{4}$ die auch von den Adressaten verstanden werden können müssen, die weder eine Hochschul- geschweige denn eine spezifisch juristische Ausbildung genossen haben;

c) schließlich wird Verstehen im Folgenden wie in der PISA-Studie der OECD ${ }^{5}$ definiert als Fähigkeit

- Informationen zu lokalisieren

- Informationen Bedeutung zu verleihen und daraus Schlüsse zu ziehen

- Verbindungen zur eigenen Situation herzustellen

Diese Definition entspricht damit übrigens in optimaler Weise der Anforderungen an juristisches Verstehen:

- Feststellen des Sachverhaltes/Tatbestandes

- Erkennen der Rechtsfolge

- Anwendung auf das eigene Rechtsproblem

2. Schrift war schon immer und ist immer noch ein elitäres Medium. Jedenfalls hat die PISA-Studie uns - in Deutschland wie in der Schweiz - schmerzhaft klar gemacht, dass $40 \%$ der Bevölkerung aus dieser Sicht $u$. a. auch als juristische Analphabeten zu betrachten sind.

Die PISA-Studie unterscheidet 5 Stufen der Lesekompetenz nebst der Kategorie „unter Kompetenzstufe 1“, d. h. eigentlich 6 Stufen. Nur Leser der Stufen 4 und 5 sind in der Lage, schwierige Leseaufgaben zu lösen. ${ }^{\circ}$ Rechtstexte sind aber - so unsere Grundannahme in III.1.a) schwierige Texte.

\footnotetext{
${ }^{4}$ Rechtstext ist hier in einem weiten Sinne zu verstehen als eine schriftliche Äußerung, der entweder von einem der beiden Adressaten oder von einem Dritten (z. B. einem Richter) eine rechtlich relevante Bedeutung zugemessen wird.

${ }^{5}$ Lernen für das Leben - Erste Ergebnisse der internationalen Schulleistungsstudie PISA 2000. Paris 2001, 398. Man mag einwenden, dass PISA die Lesekompetenzen von 15-jährigen, und nicht von i. S. des Rechts mündigen Bürgerinnen und Bürger getestet hat. Dem kann entgegengehalten werden, dass die Getesteten, die in der Schule täglich noch mit Sprachaufgaben konfrontiert werden, möglicherweise weit besser abschneiden als der Durchschnitt der Erwachsenen, der oft nur noch mündlich und in Mundart, aber weniger schriftlich und in Hochsprache kommuniziert. Man darf gespannt sein auf die Ergebnisse der Nachfolgestudie, die jetzt explizit auch die Lesekompetenz von Erwachsenen untersuchen soll. Die Datenerhebung wurde in der Schweiz erst im Juni 2003 abgeschlossen. Ältere Studien der OECD wie The International Adult Literacy Survey (IALS) zwischen 1994 und 1998 ergaben jedenfalls keine besseren Ergebnisse bezüglich der Lesefähigkeit Erwachsener.

${ }^{6}$ Leser der Stufe 3 (Lösen von Leseaufgaben mittleren Komplexitätsgrades) dürften in der Lage sein, Rechtstexte, die sie konkret betreffen (Verfügungen, Behördenschreiben),
} 
Das ergibt folgende Übersicht:

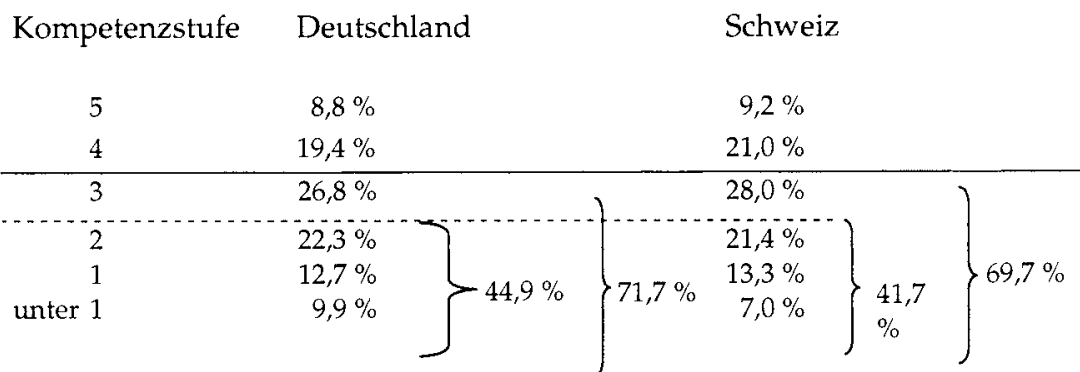

3. Die PISA-Ergebnisse sollten nun aber nicht (zumindest nicht allein) als Schreckensmeldung, sondern schlichtweg als deskriptive Bestandsaufnahme gesehen werden. Angst und Schrecken sind schlechte Ratgeber, weshalb Grund zur Befürchtung besteht, dass eine bloß oberflächliche Wahrnehmung der PISA-Resultate zu Fehlinvestitionen in der $\mathrm{Zu}$ kunft führt. Lesen - jedenfalls das Lesen und Verstehen schwieriger Texte - war immer schon eine Kulturtechnik, die nur eine kleine Elite tatsächlich beherrscht hat. Wenn es darum geht, Texte "unters breite Volk" zu bringen, ist deren Schwierigkeitsgrad zu verringern (wenn auch nicht gerade in dem Maße, wie es uns die Werbung vorexerziert). Anders gesagt: um eine Mehrheit der Bevölkerung mit Rechtstexten zu erreichen, sind diese so $\mathrm{zu}$ gestalten, dass sie von den Lesern nicht mehr Lesekompetenz erfordern als die Pisa-Stufe 3 (Lösen von Leseaufgaben mittleren Komplexitätsgrades).

4. Wie verhält sich nun aber mit der "Produktion“ von Rechtstexten? Zunächst ist - ich beziehe mich hier auf Schweizer Daten - die Zahl der berufsmäßigen Produzenten von Rechtstexten mit autoritativem Gehalt (Gesetzgebung/Rechtsprechung) verschwindend klein; in der Schweiz vermutlich weniger als $1 \%$ der Bevölkerung.

zu verstehen, $d . h$. im Sinne der hier verwendeten Verstehensdefinition die für sie relevanten Informationen zu lokalisieren, Schlüsse zu ziehen und Verbindungen zur eigenen Situation herzustellen (Vgl. oben III.1.). 


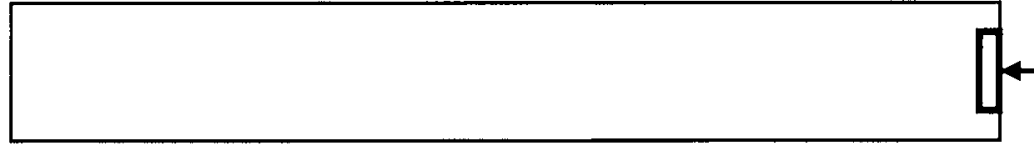

Wohnbevölkerung: $100 \%$

Anteil Mittelschüler: $20 \%$

1.400 .000

Studienabschlüsse: $15 \%$

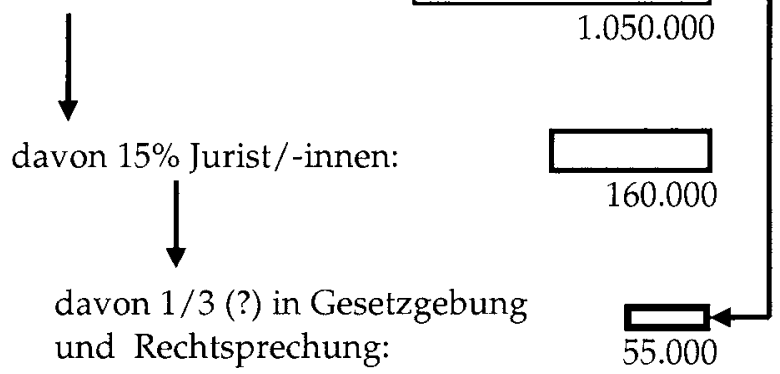

5. Ein Blick auf das Sprach-Training dieser Juristen ergibt folgendes Bild:

a) Mittelschule: Mindestens vier (4) Jahre Mittelschulunterricht, in dem gerade auch die hoch-sprachlichen Fähigkeiten gefördert werden.

b) Ein durchschnittliches Jus-Studium dauert bis zum Lizentiat fünf (5) Jahre, in denen die Studierenden intensiv in die juristische Fachsprache eingefüht werden.

c) Bis zum Abschluss einer weiteren Qualifikation (Anwaltspatent und/oder Doktorat) vergehen weitere zwei (2) oder mehr Jahre, in denen sich die jungen Juristen intensiv um die Beherrschung der Fachsprache bemühen müssen.

d) In der Praxis (am Gericht, in der Verwaltung, in der Advokatur) übt man anhand von Mustertexten und Textbausteinen, wie juristische Texte lege artis zu verfassen sind. 
e) Bis jemand in eine Position kommt, in der er maßgebend, d. h prägend auf die Formulierung von Erlassen Einfluss nehmen kann, vergehen in der Regel noch einige Jahre.

Es gibt keinen besonderen Grund für die Annahme, dass ausgerechnet Texte von derart geschulten Autoren für Nicht-Juristen - die große Mehrheit der Bevölkerung - ohne weiteres verständlich sein könnten. Hinzu kommt, dass wir es ganz offensichtlich mit einer "top-down"Produktion von Texten zu tun haben, die "bottom-up" verstanden werden sollen.

Dass Rechtstexte (zu) oft für die Bedürfnisse der Verwaltung (,topdown“) und nicht für die Bürgerinnen und Bürger („bottom-up“) geschrieben werden, hat Peter Noll schon vor dreißig Jahren diagnostiziert.7 Und offenbar ist diesbezüglich inzwischen keine merkliche Richtungsänderung erfolgt:

"Der schlimmste Feind der Verständlichkeit ist das sogenannte Verwaltungsinteresse, also der enorme Drang, in einem Regelungsbereich zu jedem denkbaren Sachverhalt eine möglichst wörtlich zutreffende Bestimmung zu formulieren." 8

6. Aber auch eine diachronische Betrachtungsweise ergibt kein anderes Bild:

Während in Juristenkreisen ohne kritische Hinterfragung die Erreichbarkeit der Adressaten über das Medium der Schrift einfach vorausgesetzt wird, zeigt eine historische Betrachtung, dass die Schrift nur auf eine sehr kurze Geschichte als Massenkommunikationsmittel zurück- und in eine sehr ungewisse Zukunft vorausblicken kann. Außerdem ist gerade die Geschichte der Alphabetisierung der Massen nicht zuletzt auch eine Geschichte der Indoktrination und der zweckgesteuerten Instruktion mit wenig demokratischer Intention.

a) Die Entwicklung der Alphabetisierung im deutschsprachigen Raum seit 1600 lässt sich in vier Phasen gliedern: Frühe Neuzeit, Aufklärung, 19. Jahrhundert, 20. Jahrhundert.

\footnotetext{
7 „Viele Gesetze sind unnötig pedantisch und ausführlich formuliert und kompliziert, enthalten unnötige und selbstverständliche Regelungen, die auf die Anwendung durch subalterne Beamte zugeschnitten sind, die in allen ihren Entscheidungen durch eine Vorschrift gedeckt sein wollen." PETER NOLL, Gesetzgebungslehre. Reinbek 1973, 166.

8 WERNER HAUCK, Warum sind unsere Gesetze so unverständlich?, in: Plädoyer 4 (2002), 27.
} 


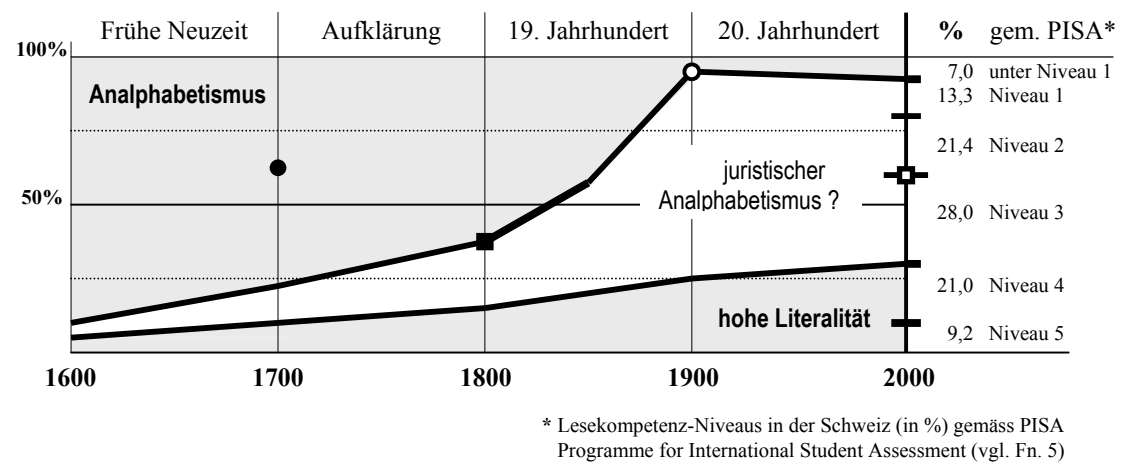

- Um 1700 sind zwei Drittel der Literatur noch in Latein.

- Bei Beginn der 1. Kodifikationswelle ist die Mehrheit der Bevölkerung nicht alphabetisiert.

- Im Höhepunkt der Alphabetisierung beginnt der Siegeszug der neuen Massenmedien.

- Über $40 \%$ der Getesteten verstehen gemäss PISA-Studie nur wenig anspruchsvolle Texte.

b) Die Frühe Neuzeit (1600-1700) ist eine Phase, in der - außerhalb gelehrter Kreise, die aber fast ausschließlich in Latein schreiben - Lesen ein gesellschaftliches Ereignis war: der Lesekundige las laut vor. "Spiegelbildlich" dazu war die Verlautbarung über den Herold oder Ausrufer die übliche Form der Promulgation behördlicher Erlasse. Alltagsnachrichten wurden über bebilderte Flugblätter, Bänkelsänger (,Zeitungslieder"), mit Mimikry und Gestik übermittelt. In den unteren Bevölkerungskreisen kam der Schrift (noch über das 18. Jahrhundert hinaus) keine Eigenständigkeit zu. „Sie war, wie die zeitgenössische Beobachtung zu Recht feststellte, ,sekundär', wurde also nicht als selbständige Übermittlung genutzt. ${ }^{\prime 9}$ Die Kultur der frühen Neuzeit ist multimedial.

Gefordert wurde - vorerst nur in den reformierten Gebieten - vor allem die Lektüre der Bibel und nur der Bibel (sola scriptura kann durchaus auch so verstanden werden), die - nebst anderen geistlichen Schrif-

\footnotetext{
${ }^{9}$ UlRICH KNOOP, Entwicklung von Literalität und Alphabetisierung in Deutschland, in: Handbiicher zur Sprach- und Kommunikationswissenschaft. Bd. 10.1. Berlin 1994, 859 ff., 866.
} 
ten (Gebetssammlungen, Psalmen, Katechismen) - fast als einzige deutschsprachige Literatur überhaupt verfügbar war. ${ }^{10}$ Ziel war die religiöse und moralische Indoktrination der Bevölkerung durch das häufige laute Vorlesen und Auswendiglernen von großen Teilen der Bibel.

c) Die Aufklärung des 18. Jahrhunderts ist eine reine top-down-Bewegung der intellektuellen Elite: ${ }^{11}$

"Die Durchsetzung der Alphabetisierung der Massen von oben (sic!) durch die Schulen geschah durch das Lesen und Auswendiglernen bestimmter Texte (Katechismus, Bibel und Teile der Bibel) - in der Absicht, die Dominanz eines traditionellen Grundwissens zu sichern. ${ }^{.12}$

Dementsprechend sollte immer laut (und wenn möglich in Gesellschaft) gelesen werden, weil nur so die angestrebte "Gedankenkontrolle" möglich war.

„Noch am Ende des 18. Jhdts. sollte die Landbevölkerung aus ihren Lesestoffen vorwiegend lernen, fromm zu denken, praktisch zu handeln, mit ihrem Los zufrieden zu sein und Befehle auszuführen. Lektüre, die zum Nachdenken anregte, war ebenso verpönt wie Lesen zur reinen Unterhaltung. ${ }^{.13}$

Indoktrination und Instruktion, die buchstäblich im Buche steht!

d) Es sind vor allem drei "theoretische Errungenschaften" der Aufklärung, welche im 19. Jahrhundert große Wirkungen entfaltet haben: die Umgestaltung der Lesetechnik bzw. des Leseverhaltens, die Orthographisierung der Schrift sowie die Einführung und Durchsetzung der allgemeinen Schulpflicht.

10 „Bis 1520 liegen in Deutschland nach Schätzungen 16,9 Mill. Bücher gedruckt vor davon sind 946.000 auf Deutsch, also $5,6 \%$, die restlichen im exklusiven Latein." KNOOP, Entwicklung von Literalität und Alphabetisierung in Deutschland (Fn. 9), 863, der an der gleichen Stelle auch darauf hinweist, dass "der Buchdruck und seine Erzeugnisse (Bücher, Flugschriften und -blätter) ... keineswegs eine unmittelbare Vermehrung des Lesen- und Schreibenlernens zur Folge hat." Um 1700 waren immer noch zwei Drittel der Bücher in Latein abgefasst.

${ }^{11}$ Jan Peters spricht bezogen auf das 18. Jahrhundert von der "Allgegenwart intellektueller Vormundschaftlichkeit" (JAN PETERS, Bäuerliches Schreiben und schriftkulturelles Umfeld. Austauschverhältnisse im 17. Jahrhundert, in: ALFRED MESSERLI/ROGER CHARTIER (Hrsg.), Lesen und Schreiben in Europa 1500-1900. Basel 2000, 87 ff.).

12 ALFRED MESSERLI, Lesen und Schreiben 1700 bis 1900 - Untersuchung zur Durchsetzung der Literalität in der Schweiz. Tübingen 2002, 502. Nach Robert Barth unterteilte sich der Bücherbesitz in den Haushaltungen im 17. und 18. Jahrhundert in folgende vier Kategorien: 1. Glaubenslehre/theologische Literatur: $50 \% ; 2$. Erbauungs-, Andachtsliteratur: $32 \%$; Moral- und Sittenlehre: $10 \%$; weltliche Literatur: $8 \%$. Quelle: ROBERT BARTH, Lesefähigkeit, Lesekulturen; 17./18. Jahrhundert. www.stub.unibe.ch/stub/vor196/02/sie. $\mathrm{html}$; Abfrage vom 23.10.2003.

${ }^{13}$ MARIE-LOUISE VON WARTBURG-AMBUHL, Alphabetisierung und Lektiire: Untersuchungen am Beispiel einer ländlichen Region im 17. und 18. Jahrhundert. Bern 1981, 190. 
Der Wechsel vom extensiven lauten Vorlesen zum intensiven lautlosen Lesen ohne Gesellschaft führt zur "Entbindung aus der multimedialen Geselligkeit, vor allem aber der Verlautbarung der Schriftlichkeit".14 Aus dem multimedialen gesellschaftlichen Vorlese-Ereignis wird eine monocodierte solitäre Fragmentierung von Leseerfahrung und Wissen.

Die Orthographisierung der Schrift wird gezielt als Mittel zur Sozialdisziplinierung entwickelt und eingesetzt (also wiederum: top-down). Sie erhält damit einen ungebührlichen Stellenwert, mit Folgen, mit denen unser Erziehungssystem heute noch zu kämpfen hat. "Die Existenz von Rechtschreibnormen kann als indirekter Faktor für die Entstehung von Analphabetismus in einigen Fällen betrachtet werden." ${ }^{15}$ Mangelhafte Orthographie ist leicht feststellbar und führt immer noch (z. B. in Bewerbungsschreiben) zu eigentlichen Diskriminierungen, ja Bloßstellungen. Bezeichnenderweise reklamierten die preußischen Schulmeister die Siege der deutschen Soldaten (1864, 1866 und 1871) als Ergebnis ihrer disziplinarischen Bemühungen. ${ }^{16}$

In der allgemeinen Schulpflicht kam also zum Stillsitzen und zur Konzentration des stillen Augenlesens die gnadenlose Selektion nach Kriterien der heiligen Orthographie. Und auch im 19. Jahrhundert war das eigentliche Ziel der allgemeinen Alphabetisierung nicht die Öffnung geistiger Räume für die Massen, sondern ihre funktionelle Anpassung an die neuen Bedürfnisse einer industrialisierten Welt. Nach wie vor war das "Romanlesen" verpönt, und das "gute" (moralisch-sittliche) Buch auch nur nach getaner Arbeit geduldet. ${ }^{17}$ Erst die massenhafte billige Verbreitung von Unterhaltungsliteratur mit Erfindung des Rota-

\footnotetext{
${ }^{14}$ KNOOP, Entwicklung von Literalität und Alphabetisierung in Deutschland (Fn. 9), 868.

${ }^{15}$ HEINZ W. GIESE, Literalität und Analphabetismus in modernen Industrieländern, in: Handbiicher zur Sprach- und Kommunikationswissenschaft. Bd. 10.1. Berlin 1994, 883 ff., 887. Schuld daran ist allerdings wohl weniger die Existenz solcher Normen als die Art ihrer Durchsetzung.

${ }^{16}$ So schreibt Messerli: „Die Toleranz der Gebildeten der unorthodoxen Schreibart der Unterschicht gegenüber scheint sich um 1800 erschöpft zu haben. Richtiges Schreiben und richtiges Verstehen gelten jetzt als Voraussetzung einer funktionierenden Kommunikation von oben nach unten" (Hervorhebung des Verf.). Siehe MESSERLI, Lesen und Schreiben 1700 bis 1900 (Fn. 12), 37. Messerli zitiert an der gleichen Stelle HaNs HEINRICH MEILI, Der schweizer'sche Briefsteller, 1810: „weil sie (die Schriftsprache) von der seinigen (jener des Volkes) so sehr verschieden ist, werden öffentliche Belehrungen, Gesetze und Verordnungen selten begriffen." Damit ist das weitere Problem der notwendigen Übersetzung von der alltäglichen schweizerischen Mundart in die deutsche Hochsprache angesprochen: die mangelnde Übung und Kompetenz im hochsprachlichen Idiom beeinträchtigt auch die Verstehensleistungen in dieser "Fremdsprache". Ähnliche Probleme zwischen Umgangs- und Hochsprache gab und gibt es natürlich auch in Deutschland.

17 Vgl. dazu MESSERLI, Lesen und Schreiben 1700 bis 1900 (Fn. 12), 161 ff.: Zeitökonomie und Auswahl.
} 
tionsdruckes (um 1870) schlägt hier eine breitere Bresche..$^{18}$ In kurzer Folge darauf werden die Erfindungen gemacht, die im 20. Jahrhundert den Niedergang der monocodierten Schriftkultur bewirken, der lange verdrängt worden ist, bzw. noch immer nicht wahrgenommen wird (nicht wahrgenommen werden will).

e) Zu Beginn des 20. Jahrhunderts galt die Massenalphabetisierung als erfolgreich abgeschlossen. Ab 1912 verzichtete das Deutsche Reich darauf, den Aspekt der Alphabetisiertheit in den Volkszählungen zu berücksichtigen. Im gleichen Jahr wurde in England mit der BBC die erste kommerzielle Rundfunkanstalt gegründet. Zu diesem Zeitpunkt hatten sich Photographie, Phonographie und Film bereits definitiv als weitere neue Massenmedien etabliert. ${ }^{19}$ Hatte die Schrift das 19. Jahrhundert (mangels Konkurrenz und mit Unterstützung der "sola scriptura"-Doktrin) noch weitgehend beherrscht, standen im Zeitpunkt des Höhepunktes ihrer Macht (1912) diejenigen Medien bereit $-z$. T. schon in voller Blüte (wie das Kino) -, welche ihr im 20. Jahrhundert den Rang ablaufen sollten.

Hoch gerechnet hat die Herrschaft des elitären Schriftcodes (topdown) höchstens hundert Jahre (von ca. 1850 bis 1950) gedauert. Spätestens mit der flächendeckenden Versorgung der Bevölkerung mit Radio- und Fernsehempfängern (nach 1950) ist die (westliche) Gesellschaft zur Multimedialität zurückgekehrt. Und ständig kommen neue Medien hinzu: das flächendeckende Telefonnetz führt ab 1960 zur neuen, so genannten zweiten Mündlichkeit, ${ }^{20}$ welche mit den allgemein verwendeten Mobiltelefonen ( $a b$ 2000) nochmals einen Bedeutungszuwachs erfahren hat. Hinzu kommen weitere Kommunikationsformen wie Internet, E-Mail, SMS, MMS, die zwar auch, aber eben nicht mehr allein schriftbasiert (und zudem oft nicht hochsprachlich), sondern (mit Bildern und Tönen) multicodiert sind.

\footnotetext{
${ }^{18}$ Gegen Ende des 18. Jahrhunderts schon setzte die so genannte Lesesucht-Debatte ein, nachdem erstmals ein größeres Angebot an Unterhaltungsliteratur auf den Markt kam; vgl. MESSERLI, Lesen und Schreiben 1700 bis 1900 (Fn. 12), 130 ff. Es dürfte kein Zufall sein, dass diese Debatte am Ende des 19. Jahrhunderts neue Höhepunkte erreichte, weil eben die "fragwürdigen" Bücher nun auch billiger wurden und so viel weitere Kreise der nun fast vollständig des Lesens (einfacherer Texte) kundigen Bevölkerung erreichten.

191869 Rotationsdruckverfahren; 1877 Edisons Phonograph; 1884 Eastmans Rollfilm; 1891 Edisons Kinetoskop; 1895 erste kommerzielle Kinovorführung; 1906 erste Rundfunksendung; 1911 erste Übertragung eines Fernsehbildes. Vgl. auch FRANZ-JOSEF JELICH, Arbeiterkultur - Massenkultur. Neue Medien und die Erosion der Arbeiterkultur, in: GeRd HuRLE/FRANZ-JOSEF Jelich (Hrsg.), Vom Buchdruck in den Cyberspace? Marburg 1995, $92 \mathrm{ff}$.

20 Vgl. HEINZ SCHLAfFER in der Einleitung zu JACK GOODY/IAN WATt/KatHLEEN GOUGH, Entstehung und Folgen der Schriftkultur. Frankfurt am Main 1981, 7.
} 
7. Aus juristischer Sicht verdienen die beiden Jahrhundertwenden um 1800 und um 1900 besondere Aufmerksamkeit.

a) Um 1800 erfolgt die erste Kodifikationswelle: 1794 das Preußische Allgemeine Landrecht, 1804 der Code civil, 1811 das Allgemeine Bürgerliche Gesetzbuch. Die schriftliche Fixierung des geltenden Rechts erfolgt ganz offensichtlich im ",aufklärerischen Kontext", top-down, in einer Zeit, zu der die weitaus größte Mehrheit der Bevölkerung weder Lesen noch Schreiben konnte. ${ }^{21}$ Hinzu kommt, dass diese Texte - selbst aus der Optik ihrer Verfasser - auch für die Minderheit der Lesekundigen nicht verständlich waren. Schon 1721 hatte Christian Wolff vorgeschlagen, die

„Statute doppelt zu verfertigen, einmal zum täglichen Gebrauch ohne einige Erläuterungen, darnach zum besseren Verstande derselben mit beigefügten Gründen und sonst nötiger Ausführung. ${ }^{22}$

1788 nahm Svarez, einer der Redaktoren des Preußischen Allgemeinen Landrechts, den Gedanken wieder auf, da „wir ein doppeltes Gesetzbuch nötig haben: eines für den Richter und Rechtsgelehrten und das andere für das Volk überhaupt." 23

Mit der Inkraftsetzung der neuen Kodifikationen wurde zunächst einmal die große Mehrheit der illiteraten Bevölkerung mit einem schriftlichen Gesetz konfrontiert, das selbst die Minderheit der Lesekundigen kaum verstehen konnte; $d$. h. die Klasse der ,juristischen Analphabeten" war entstanden. Die Alphabetisierungsanstrengungen des 19. Jahrhunderts (in der Schweiz steht die allgemeine Schulpflicht erst seit 1848 in der Bundesverfassung, die kantonalen Schulordnungen sehen seit ca. 1830 eine allgemeine Schulpflicht vor) dürften trotz der abnehmenden Anzahl völlig illiterater Menschen wenig beigetragen

21 Und wenn, nur mit sehr begrenzter Kompetenz (vgl. oben III.6 b und c), wobei es natürlich große Unterschiede gab, insbesondere zwischen städtischen und ländlichen, katholischen und reformierten Gegenden sowie zwischen den verschiedenen Niveaus: Lesen von Gedrucktem, Lesen von Handschriften, und nochmals ganz unterschiedlich je nach Geschlecht und Milieu.

22 CHRISTIAN WOLFF, Vernünftige Gedanken von dem gesellschaftlichen Leben der Menschen, zitiert nach HARALD KINDERMANN, Gesetzessprache und Akzeptanz der Norm, in: THEO ÖHLINGER (Hrsg.), Recht und Sprache. Wien 1986, 59.

23 CARL GOTTLIEB SVAREZ, Inwiefern können und müssen Gesetze kurz sein?, zitiert nach KINDERMANN, Gesetzessprache und Akzeptanz der Norm (Fn. 22), 66. Der Gedanke der Gesetzeserläuterung findet sich im Übrigen schon bei PLATON, Nomoi, 4. Buch (718b ff.), wo er den guten Gesetzgeber mit dem guten Arzt vergleicht, der seine Behandlung mit seinem Patienten bespricht und so "auf doppeltem Wege" die Heilung bewirkt (720 e). 
haben, die „strukturellen" Schwächen dieser Erlasse, nämlich ihre schon von den Verfassern erkannte Unverständlichkeit für Laien, zu beheben.

b) Die Ausgangslage für die zweite Kodifikationswelle um 1900 (1900: BGB; 1912: ZGB) war günstiger: Zum einen erfolgte die Verschriftlichung hier auf dem Höhepunkt der Massenalphabetisierung und zum andern hatte zumindest Eugen Huber erkannt, dass die moderne Gesetzgebung versuchen müsste,

„sich einer Entwicklung zu erwehren, die auf dem Boden der Begriffsjurisprudenz mit Notwendigkeit die Volkstümlichkeit ausschliessen möchte. ... Sie (die Rechtssprache) ist nicht an das tatsächlich unmögliche und technisch wertlose Requisit der Fassung der Rechtssätze in abstrakter Gestalt, gleich als ob sie konkret angewendete Rechtssätze wären, gebunden, sondern bewegt sich zur Bewältigung ihrer grossen Aufgaben frei, volkstümlich, reich im Besitz aller Kräfte, die im Rechtsleben mitzuspielen berufen sind ..."24

Allerdings erkaufte sich das ZGB seine höhere Verständlichkeit nur um den Preis einer größeren Lückenhaftigkeit und Unvollständigkeit.25 Oder kurz: Der Leser des ZGB versteht den Gesetzestext weit besser als jener des BGB, dennoch weiß er über das geltende Recht nicht mehr, weil der Schweizerische Gesetzestext nur zusammen mit der Rechtsprechung ein zuverlässiges Bild über die Rechtslage ergibt. ${ }^{26}$ Die Rechtsprechung sowie die dazugehörige reiche Literatur („bewährte Lehre und Überlieferung", Art. 1 Abs. 3 ZGB) ist den Rechtslaien in der Regel weder physisch, fachlich noch sprachlich zugänglich.

Damit ist ein Kardinalproblem angesprochen, welches selbst ein mustergültig verständlich formuliertes Gesetz nicht lösen kann: die Bürgerin, der Bürger erfährt auch daraus nicht, was in seiner Situation nun rechtens ist. Auch der (verstandene) Wortlaut des Gesetzestextes ist nur Einstieg in das Verstehen des Rechts. ${ }^{27}$ Aber immerhin: ein verständlicher Text erlaubt einen besseren Einstieg, und - was oft schon sehr weit hilft - eine Einschätzung der Grundwertungen des Gesetzgebers und damit des Bereiches, in welchem eine konkrete Entscheidung

24 Eugen Huber, Das Absolute im Recht. Bern 1922, 59.

${ }^{25} \mathrm{Vgl}$. dazu RUDOLF GMUR, Das Schweizerische Zivilgesetzbuch verglichen mit dem Deutschen Bürgerlichen Gesetzbuch. Bern 1965, 28 ff. (zu Stil und Sprache des BGB), 40 ff. (zum volkstümlichen Charakter des ZGB).

${ }^{26}$ Das gilt natürlich auch für das BGB, aber nach Gmür kann vom schweizerischen Richter - wegen der Unschärfen des volkstümlichen Gesetzes - „eine gleichmässige und vorausberechenbare Rechtsprechung kaum im gleichen Ausmass erwartet werden wie vom deutschen Richter"; siehe GMÜ, Das Schweizerische Zivilgesetzbuch verglichen mit dem Deutschen Bürgerlichen Gesetzbuch (Fn. 25), 42.

27 DAVID DÜRR, Zürcher Kommentar zum ZGB. Zürich 1998, Vorbemerkungen zu Art. 1 und 4, N $226 \mathrm{ff} .:$ „Die Einstiegsfunktion des Wortlauts zeigt, dass es beim rein sprachlichen Verstehen nicht bleiben kann. Auch der klare Wortlaut ist bloss Einstieg." ( $\mathrm{N}$ 228). 
vermutlich liegen wird. Zudem ist ein verständlich abgefasstes Gesetz auch für die Fachleute und erst recht für nicht spezialisierte Juristen das bessere Arbeitsinstrument. Viele Konflikte über die Bedeutung einer Norm müssen nur deswegen - und zwar oft unter gleichermaßen hoch qualifizierten Spezialisten - ausgetragen werden, weil der Gesetzestext - je nach Adressat - nicht (nie) verständlich (genug) ist.

\section{Bilder, um besser im Bilde zu sein}

1. Wer etwas verstanden hat, sagt oft: „Ich bin im Bilde", d. h. er sieht sozusagen vor seinem geistigen Auge, was ihm ein anderer vermitteln wollte. Man ist mit Sicherheit besser im Bilde, wenn dazu noch ein verbaler (mündlicher oder schriftlicher) Begleittext zur Verfügung steht.

Das hängt damit zusammen, dass wir Menschen mulitimediale Wesen sind. Natürliche Kommunikation verläuft immer multimedial. Eine $\left(\right.$ fast $\left.^{28}\right)$ monocodierte Kommunikation wie die Schrift - Hauptträger rechtlicher Kommunikation - ist eine kulturgeschichtliche Erscheinung, deren Reichweite immer auf kleine Eliten begrenzt war, jedenfalls bezüglich der Beherrschung schwieriger Texte.

2. Seit den Arbeiten von Paivio gibt es eine breit angelegte kognitionswissenschaftliche Forschung, welche die multimediale Informationsaufnahme und -verarbeitung durch uns Menschen belegt. ${ }^{29}$ Gut belegt ist inzwischen auch die besondere Leistungsfähigkeit des Duos BildSchrift innerhalb der gesamten multicodiert verlaufenden Kommunikation. Die folgenden drei Aussagen $(a-c)$ dürfen als in der kognitions-/ kommunikationstheoretischen Lehre weitgehend unbestritten gelten:

a) There is nothing better than a picture for making you think of questions you had forgotten to ask (even mentally). 30

Tukey bringt damit eine der wichtigsten Qualitäten von Bildern ${ }^{31}$ auf den Punkt: Bildliche Darstellungen gedanklicher Inhalte sind oft besser

\footnotetext{
${ }^{28}$ Genau besehen ist natürlich auch Schrift mehrfach codiert: Hand- oder Maschinenschrift, Schrifttype/-größe, Trägermaterial (Notizzettel oder parfümiertes Briefpapier) etc. Rechtstexte (u. a. Gesetzestexte) sind aber auch in dieser Hinsicht standardisiert gerade mit dem Ziel, den Inhalt möglichst neutral zu übermitteln.

29 Allan Paivio, Mental Representations, A Dual Coding Approach. Oxford 1986.

30 JOHN W. TUKEY/PAUL A. TUKEY, Computer Graphics and Exploratory Data Analysis, in: William Cleveland, The Collected Works of John W. Tukey. Bd. 5: Graphics 19651985. New York 1988, 421.

${ }^{31}$ Zum hier verwendeten Bildbegriff vgl. unten V.1.
} 
geeignet als mühsame Verbalisierungsversuche, diese Inhalte dem "Hersteller" des Bildes wie seinen Adressaten klar zu machen.

b) Two codes are better than one ....

... kann als Quintessenz von breit angelegten empirischen Untersuchungen über die Verbesserung von Verstehens- und Behaltensleistungen bei einer dual-codierten Informationsvermittlung (gegenüber bloß schriftlich codierter) gelten.

c) ... you should use both..$^{33}$

... ist schließlich die Empfehlung des Neurologen nach Ausführungen über die multimediale Informationsverarbeitung in unserem Gehirn.

3. Bilder können zugegebenermaßen auch verwirren oder Unklarheit stiften. Deshalb muss erfolgreiches Dual-coding mit eigens für die Zwecke juristischer Kommunikation entwickelten und auf den begleitenden („kommunizierenden“) Schriftcode abgestimmten Bildern erfolgen. Darüber, wie solche Bilder zu gestalten wären, weiß man heute schon sehr viel. Zu erwähnen sind hier zunächst die empirisch breit abgesicherten Gesetze der Gestaltpsychologie; ${ }^{34}$ besondere Beachtung verdienen auch die von Ramachandran/Hirstein aus neurologischer Sicht vorgeschlagenen acht "Design-Gesetze“ ${ }^{.35}$

$\mathrm{Zu}$ warnen ist allerdings davor, „Rechtsbilder" mit Versatzstücken aus der Rechtsikonologie herstellen zu wollen. ${ }^{36}$ Dieser Ansatz setzt seinerseits wiederum eine lange rechtsikonographische Schulung voraus vergleichbar dem Schrifttraining der Juristen -, statt bei den "natürlichen" Kommunikationsfähigkeiten und -strategien multimedial begabter Menschen anzusetzen. Außerdem haben selbstverständlich auch Bilder ihre Grenzen: Ohne Vorstellung darüber, welche Bilder sich wofür eignen, ist keine erfolgreiche Dual-Codierung der Rechtskommunikation denkbar.

32 RAYMOND W. Kulhavy/William A. StOCK/Linda C. CATERiNO: Reference Maps as Framework for Remembering Texts, in: WOLFGANG SCHNOTZ/RAYMOND W. KULHAVY (Hrsg.), Comprehension of Graphics. Amsterdam 1994, 155.

${ }^{33}$ JOHN J. RATEY, A User's Guide to the Brain. New York 2001, 281.

34 Insbesondere die Gesetze der Nähe, der Ähnlichkeit, der guten Gestalt, der guten Fortsetzung und des gemeinsamen Schicksals.

35 VILAYNUR RAMACHANDRAN/WiLliam HiRSTEIn, The Science of Art: A neurological Theory of aesthetic experience, in: art and the brain. Journal of Consciousness Studies 6 (1999), 15-51.

36 So aber der Ansatz von COLETTE BRUNSCHWIG, Visualisierung von Rechtsnormen. Zürich 2001. 
Umgekehrt aber darf aufgrund der heute vorliegenden kognitionswissenschaftlichen Erkenntnisse behauptet werden, dass eine sorgfältig aufeinander abgestimmte Bild-Text-Kommunikation jeder nur monocodierten Informationsübermittlung überlegen ist, und zwar sowohl bezüglich der Verstehens- als auch der Behaltensleistungen.

Diese bessere Verständlichkeit von multimedial-gestalteten Gesetzen gefährdet allerdings das Monopol der „schriftgelehrten" Juristen, das sich dank der top-down-Organisation dieser Texte leichter verteidigen lässt, als wenn das Recht in geeigneter Form visualisiert wird:

„Law presented by visual means will likely be more understandable and accessible - not only to persons with limited textual skills, or persons with a limited ability to interpret legal verbiage, but to the public as a whole. In a more imaginistic environment, lawyers may find not only that they retain less of monopoly on legal information, but that they have less social power as the verbal base of their authority is eroded." ${ }^{\prime 37}$

\section{Was aber ist ein Bild?}

1. Was ist ein Bild? Es gibt mindestens so viele Bild-Definitionen wie Theoretiker des Visuellen. Selbst statische Darstellungen auf zweidimensionalen Ebenen lassen keine scharfen Abgrenzungen zu (vgl. Elkins, zitiert in Fn. 1). Für unsere Zwecke - Fragen der optimalen Vermittlung von Informationen über das Recht - beschränken wir uns auf einige wenige "technische" Aspekte. Im Spektrum zwischen Hören und Sehen, zeitlich und räumlich bzw. seriell oder parallel, monocodierter oder multicodierter Information lassen sich Schrift und Bild wie folgt einordnen:

\footnotetext{
37 BERNARD J. HIBBITS, The Re-vision of Law: The Pictoral Turn in American Legal Culture.
} Paper delivered to the Annual Meeting of the College Art Association. Boston 1996. 


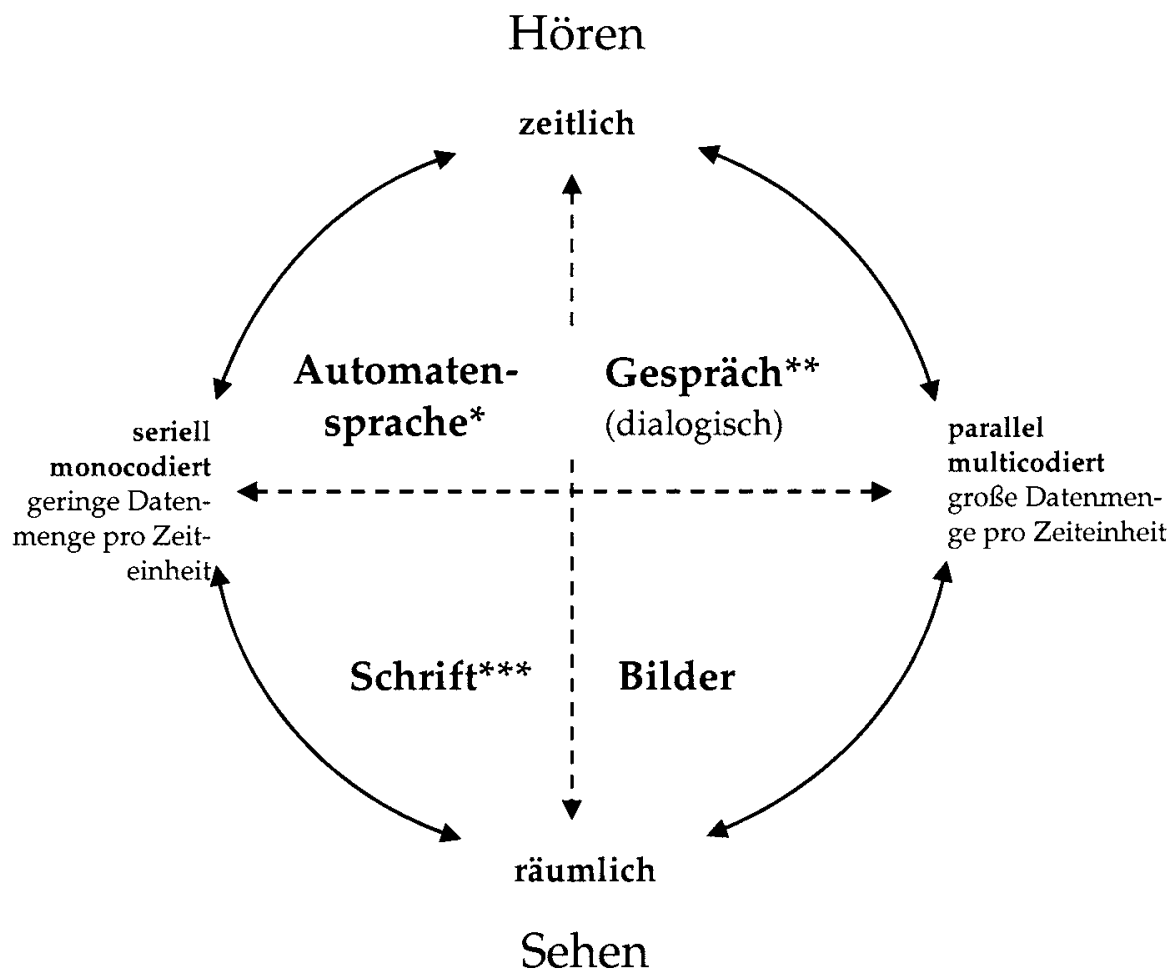

* $\quad$ Beispiel: Die sprechende Uhr. Auch sie ist nie wirklich nur monocodiert, wird aber so wahrgenommen. Eine Stimmmodulation wird nicht erwartet, ist gar nicht erwünscht.

** Jedes Gespräch - auch am Telefon - ist dialogisch und multicodiert bzw. durch nicht-verbale Codes (Gestik, Mimik, Stimmmodulation, Pausen etc.) strukturiert.

*** Auch Schrift ist genau besehen multicodiert (Wahl von Papier, Schriftart, Schriftgrad etc.). Bei Gesetzestexten spielt die einheitlich normierte Typografie aber kaum eine eigene Rolle (wie z. B. die Kalligrafie im Liebesbrief auf parfümiertem Büttenpapier).

Bilder sind demzufolge visuelle Informationsangebote, bei denen eine hohe Datenmenge parallel und multicodiert räumlich präsentiert wird. Anders als bei der seriell organisierten Schrift ist keine Wahrnehmungsreihenfolge vorgegeben, was nicht ausschließt, dass eine Wahrnehmungssteuerung auch bei Bildern möglich ist (durch sogenannte eye-catcher). Nicht eingegangen wird hier auf die Besonderheiten beweglicher und/oder interaktiver Bilder; d. h. im Folgenden sind immer nur statische Bilder gemeint. 
2. Arten von Bildern: Wir können Bilder nach ihrem Abstraktionsgrad klassieren in darstellende Bilder, Schemata und logische Bilder:

\section{Darstellendes Bild \\ (, ,ikonische“ Abstraktion) \\ (möglichst) nicht arbiträre Wiedergabe von Realitäts-} ausschnitten

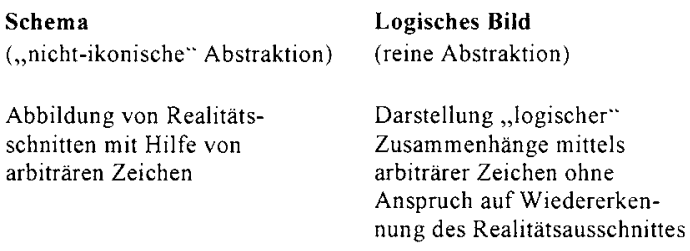

Abbildung von RealitätsDarstellung "logischer Zusammenhänge mittels arbiträrer Zeichen ohne Anspruch auf Wiedererkennung des Realitätsausschnittes
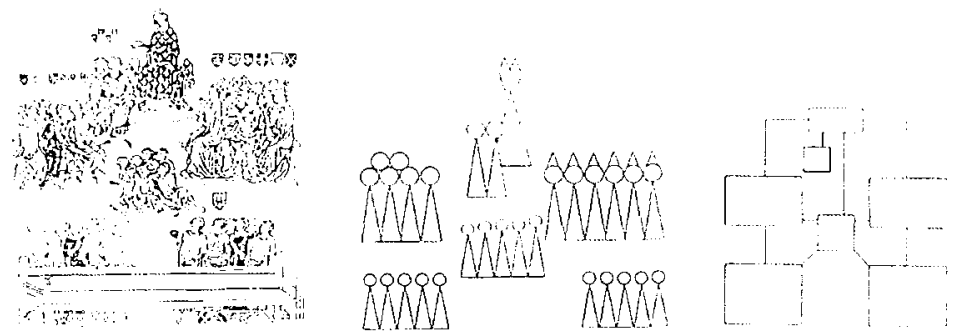

Mit oder dank ihrer hohen Abstraktion eignen sich Schemata und logische Bilder natürlich auch besonders gut, abstrakte Beziehungen darzustellen, sei das eine hierarchische Ordnung (wie im vorstehenden Beispiel) oder aber z. B. Verwandtschaftsbeziehungen (wie im Test unter Ziffer 2).

3. Was können Bilder besser - wo sagt ein Bild mehr als tausend Worte? Sachgerecht - d. h. auf die visuellen Fähigkeiten der Adressaten abgestimmt - konstruierte Bilder eignen sich hervorragend, bei großen Datenmengen Übersicht und Ordnung zu schaffen und damit auch die Suche nach Informationen (z. B. nach einer Behörde) zu erleichtern. Die konsequente grafische Darstellung hilft aber auch zu vermeiden, dass Dinge vergessen gehen ("think of questions you had forgotten to ask"), oder dass Lücken und Widersprüche unentdeckt bleiben. Ganz besonders gut eignen sich Bilder aber zur Darstellung von Strukturen (z. B. Gerichtsorganisation) oder Abläufen (Verfahrensdiagramme).

„Drückt man seine Gedanken in graphischen Darstellungen aus, so wird man gezwungen, die Gedanken möglichst klar zu formulieren. Unklarhei- 
ten, Lücken, verborgene Gedankengänge und Widersprüche werden sichtbar. ${ }^{\prime 38}$

4. Dass Bilder hervorragend geeignet sind, bei großen Datenmengen und/oder schwierigen Verhältnissen Klarheit zu schaffen, weiß „das Recht" eigentlich schon lange. Der schweizerische Gesetzgeber fordert dementsprechend auch von seinen "Untertanen" (die top-down-Attitude), dass sie ihrerseits gefälligst Bilder zu verwenden haben, um eine Haftpflicht $z u$ vermeiden oder einen Rechtsanspruch zu erlangen.

a) Aufgrund der strengen Rechtsprechung zur ärztlichen Aufklärungspflicht wird heute kein Chirurg mehr einen schwierigeren Eingriff vornehmen (Notfälle vorbehalten), ohne zusammen mit seinem Patienten das Formular ,Aufklärungsprotokoll/Einwilligung" (der Schweizerischen Gesellschaft für Chirurgie) auszufüllen. ${ }^{39}$ Fast die Hälfte der ersten Seite ist reserviert für eine Skizze der Operation, während auf Seite zwei als weitere Hilfsmittel für die Aufklärung Bilder/Foto, Broschüre, Modelle, PC-Programme erwähnt werden. Zudem wird bei weiteren Punkten (möglichen Operationserweiterungen, möglichen Komplikationen) empfohlen, diese schon in der Operationsskizze anzumerken.

b) Wer in der Schweiz einen Geldspielautomaten aufstellen will, hat u. a. folgende Unterlagen einzureichen: Fotografie des Geldspielautomaten, Zeichnungen und Pläne ${ }^{40}$ des Geldspielautomaten sowie seiner Komponenten und Bauteile sowie Schemata und Ablaufdiagramme. ${ }^{41}$

c) Art. 28 der Patentverordnung ${ }^{42}$ schreibt genau vor, wie die Zeichnungen auszugestalten und welche mit einer Patentanmeldung einzureichen sind: sie müssen $z$. B. "in unverwischbaren, gleichmässig starken und klaren Linien und Strichen ohne Farben oder Tönungen" ausgeführt werden (Abs. 2) und gewährleisten, "dass die fotografische oder elektronische Wiedergabe alle Einzelheiten mühelos erkennen lässt" (Abs. 4). Das Verbot, Farben zu verwenden, ist hier offensichtlich von den begrenzten Reproduktionsmöglichkeiten der Verwaltung (also topdown) diktiert, wobei sich Farben - gerade in technischen Zeichnungen

\footnotetext{
38 FRIEDRICH LACHMEYER, Zur graphischen Darstellung des Rechts, in: Österreichisches Anwaltsblatt 1976, 263.

${ }^{39}$ Vgl. BGE 117 Ib 197.

40 Welchen Unterschied der Gesetzgeber hier zwischen (technischen) Zeichnungen und Plänen sieht, wird aus dem Kontext nicht klar.

${ }^{41}$ Verordnung des EJPD über Überwachungssysteme und Glücksspiele vom 20. Dezember 2001 (SR 935.521.21)

42 Verordnung über die Erfindungspatente vom 19. Oktober 1977 (SR 232.41).
} 
- hervorragend eignen würden, verschiedene Details (z. B. getrennte Stromkreise) klar voneinander abzugrenzen. ${ }^{43}$

d) Die zwingend vorgeschriebenen Gefahrenhinweise auf Spielzeugen müssen in den drei Amtssprachen abgefasst sein. ,Sie können durch international gebräuchliche Piktogramme ersetzt werden" ${ }^{44}$ was in vielen Fällen sinnvollerweise auch gemacht wird. Damit kommt diesen Bildern die Qualität einer Rechtsquelle zu. ${ }^{45}$

\section{Das Adressatenproblem}

1. Wie beim Textverständnis stellt sich auch bei der Bildverwendung das Adressatenproblem. Klar zu unterscheiden sind folgende Verwendungssituationen:

a) rechtstheoretische

(i) didaktische

(ii) wissenschaftliche

Die Anforderungen an Bilder sind in didaktischen Zusammenhängen (Scriptum, Lehrbuch) andere als in einem wissenschaftlichen Kontext (Monographie zu einem Spezialthema von und für Spezialisten). Es ist selbstverständlich, dass in einem Scriptum für Studienanfänger andere (einfachere) Gestaltungsprinzipien anzuwenden sind als in einer Grafik für eine hochwissenschaftliche Diskussion; in Letzterer dürfen durchaus auch einmal komplizierte, auf den ersten Blick (und für den nicht spezialisierten Betrachter) nicht sofort „einsichtige" Bilder verwendet werden.

b) rechtspraktische

(i) Klienteninformation

(ii) Rechtsschrift (Vertrag, Klage, Urteil, Verfügung etc.)

(iii) Rechtssetzungsprojekte, -entwürfe

(iv) Erlasse

43 Verwendung von Farben in der Gesetzgebung wirft allerdings andere Probleme auf, die hier nicht abgehandelt werden können.

${ }^{44}$ Verordnung des EDI über die Sicherheit von Spielzeug vom 27. März 2002 (SR 817.044.1) Anhang 3 II 1c und 2a.

${ }^{45}$ Nach Bühler sind Bildzeichen oder Symbole, die ein Hersteller auf Maschinen anbringt, um die Betreiber auf allfällige Gefahren aufmerksam zu machen, keine Rechtsquelle. Allerdings hat er auch schon vorausgesehen, "dass sie einst $z$ u solchen werden" eben z. B. die Gefahrenpiktogramme gemäß Spielzeugverordnung; siehe THEODOR BÜHLER, Rechtserzeugung, Rechtserfragung, Legitimität der Rechtsquellen. Zürich 1985, 178 f. 
Der heuristische Wert einer Zeichnung in der Instruktion durch und Information des Klienten dürfte jedem Anwalt geläufig sein. Noch eher selten erscheinen Bilder in Rechtsschriften, wobei zudem nach Herstellern (Private versus Behörden) zu differenzieren ist. Immerhin gibt es große Rechtsbereiche ( $\mathrm{z}$. B. das Planungs- und Baurecht ${ }^{46}$ ), in welchen die Zeichnung schon längst zum integrierenden Bestandteil der rechtlich relevanten Normen und/oder Verträge gehört.

Dass Behörden (z. B. Gerichte) in ihren Rechtsschriften (Entscheidungen) Bilder verwenden, ist immer noch eine sehr seltene Ausnahme..$^{47}$ In beiden Fällen - Verwendung durch Private wie Behörden - bestehen offensichtliche Unsicherheiten bezüglich der Gestaltung wie auch der Wirkung und Folgen solcher Bilder. Das ist nur zu verständlich, wenn man den mangels adäquater Schulung weit verbreiteten BildAnalphabetismus ${ }^{48}$ in Betracht zieht. In Gesetzgebungsprojekten sind Bilder dagegen relativ häufig anzutreffen, allerdings oft in gestalterisch sehr schlechter Qualität, die bis zum Konflikt, ja völligem Widerspruch zwischen Text und Bild gehen kann. ${ }^{9}$ Schlimm daran ist vor allem, dass derart schlechte Bilder von den „Bildgegnern" natürlich noch so gerne als Argument für die Untauglichkeit von Bildern im Recht missbraucht werden können.

In Erlassen selbst sind Bilder dagegen wieder Raritäten, wenn man von den Verkehrszeichen und den zahlreichen technischen Verwaltungsvorschriften (mit Tabellen, Diagrammen, Plänen etc.) absieht. Organigramme für Behördenstrukturen fehlen in den Erlassen immer noch. Auch hier schlägt der schon erwähnte Bild-Analphabetismus offensichtlich voll durch.

2. Es gibt eigentlich nur einen Bereich im Recht, in dem sich Bilder durchgesetzt haben: die Verkehrszeichen sind verbindliche Kurzfassungen ganzer Paragraphen des Straßenverkehrsrechtes. Sie haben sich

${ }^{46}$ Vgl. z.B. die Art. 4, 6, 8-12 des schweizerischen Raumplanungsgesetzes vom 22. Juni 1979 (SR 700).

${ }^{47}$ Vgl. den Entscheid des Bundesgerichtes vom 3.10.2002, in welchem zur Klärung der Frage des Vortrittsrechtes auf Straßenverzweigungen eine Zeichnung verwendet wurde (BGE 129 IV 45).

${ }^{48}$ Vgl. dazu CHRISTIAN DOELKER, Ein Bild ist mehr als ein Bild. Stuttgart 1997, 16 ff. (Bilderverbot und Bilderverachtung).

${ }^{49}$ Ein Beispiel dafür findet sich im BBL 2002, 5011 (Bericht des Bundesrates und Botschaft über die Änderung des Postorganisationsgesetzes vom 22. Mai 2002). Unter dem Titel "Vergleich Laufzeiten im nationalen Briefpostverkehr" wird auf Seite 5024 eine Grafik gezeigt, die suggeriert, dass die Postlaufzeiten in der Schweiz am längsten seien, wobei aber genau das Gegenteil ausgesagt werden sollte. 
nicht nur national, sondern praktisch weltweit (mit erstaunlich geringfügigen Abweichungen) bewährt.

Alle anderen Bereiche des Rechts sind aber nach wie vor eine Buchstabenwüste der Schrift. Weder in der Rechtsdidaktik noch in den Gesetzgebungsarbeiten, geschweige denn in den Gesetzen, gibt es eine systematische, regelgeleitete Bildkonstruktion oder Bildverwendung.

Was Raphaela Henze in Deutschland festgestellt hat, gilt bezüglich der Rechtsdidaktik weitgehend auch für die Schweiz: „,... ein pictoral turn hat in der juristischen Ausbildung nicht stattgefunden." 50 "Lehrmittel für Studierende" ohne jegliche Illustration sind nicht der Ausnahme-, sondern eher der Regelfall und tragen mit ihrer reduktionistischen "Monocode-Didaktik" mit dazu bei, eine bessere (,personelle") Ausgangslage für die erwünschte und nötige bessere Verständlichkeit von Rechtstexten von vorneherein zu verhindern (vgl. oben II.).

"It's raining images outside, but we are locked indoors. ${ }^{51}$ Will die Rechtswissenschaft nicht zur hermetischen Geheimwissenschaft verkümmern, wird sie nicht darum herumkommen, in den Bilderregen. hinauszutreten - und sich davon erfrischen zu lassen.

\footnotetext{
${ }^{50}$ RaPHAELA HenZe, Visuelle Darstellung im Hochschulunterricht. Arbeitspapier für die Tagung Kommunikative Funktionen des Bildgebrauchs im Recht. Bochum 2001, 11; DIES., Bildmedien im juristischen Unterricht. Diss. Berlin 2003

${ }^{51}$ BARBARA STAFFORD, Good Looking/Essays on the Virtue of Images. Boston 1996, 66.
} 